\title{
Crecimiento y productividad de las ramas de servicios El papel de las TIC
}

\section{Andrés Maroto Sánchez ${ }^{\text {a }}$}

Universidad Autónoma de Madrid

\section{RESUMEN}

Este trabajo analiza empíricamente la contribución de las TIC al crecimiento de la productividad desde 1980 hasta la actualidad para un conjunto de países de la OCDE. La fuente estadística escogida son las series recientemente publicadas en la base EUKLEMS, debido a su amplia desagregación sectorial, lo que favorece el análisis aplicado del sector servicios y sus principales ramas de actividad.

Tras una revisión bibliográfica de las relaciones existentes entre crecimiento, productividad y capital tecnológico; se cuantifica el comportamiento de la productividad analizando con especial atención el papel desempeñado por las TIC y su contribución al crecimiento económico. Finalmente, se realiza un análisis econométrico de las relaciones anteriormente descritas. El principal valor añadido de este trabajo con respecto a la amplia literatura existente es que estas técnicas se aplican al caso del sector servicios, comparándolo con las manufacturas, y, especialmente, desagregando las diferencias intrasectoriales que caracterizan un sector tan heterogéneo como el terciario.

El conjunto de evidencias tiende a refutar la tesis tradicional sobre la baja contribución de los servicios al crecimiento agregado de la productividad. El sector en conjunto presenta crecimientos de la productividad modestos, en relación con otros sectores

a Profesor Ayudante Doctor de la Universidad Autónoma de Madrid e Investigador del Instituto de Análisis Económico y Social (IAES). C/ Francisco Tomás y Valiente, 5; 28049 Cantoblanco (Madrid). Tfo: 914972955. Fax: 914976930. E-mail andres.maroto@uam.es

Recibido: septiembre de 2010. Aceptado: octubre de 2010. 
económicos. Pero este resultado se matiza o limita cuando el análisis se hace de forma desagregada.

Palabras clave: TIC, Productividad, Contabilidad del crecimiento, Sector servicios Clasifición JEL: C33, J24, L80, O47

\begin{abstract}
This paper empirically analyzes the contribution of ICT to productivity growth since 1980 onwards of a sample of OECD countries. The series have been obtained from the EU KLEMS database, with a deep industrial disaggregation which it is an advantage to analyze the service sector and its main activity branches.

After a survey of the literature on the relationships among economic growth, productivity and technological capital, using a growth accounting framework, they are assessed the productivity patterns, focusing on the role of ICT capital and its contribution to economic growth. Finally, an econometric model is implemented to test those relationships. The value added of this paper is that this methodology is applied to the service sector, comparing to the patters observed in manufacturing industries, and, principally, disaggregating the intra-industrial differences which characterize the tertiary sector.

Results partially refute the traditional hypothesis of a low productivity in services. The sector as a whole present lower productivity growth rates than other main economic sectors, such as manufacturing. However, when disaggregating our analysis some tertiary industries show dynamic behaviours.
\end{abstract}

Key words: ICT, Productivity, Growth accounting, Service sector

JEL Clasiffication: C33, J24, L80, O47 


\section{INTRODUCCIÓN}

El lento crecimiento de la productividad desde un punto de vista tanto histórico como internacional ha sido centro de debate en las economías europeas, particularmente la española, en los últimos años. Una de las causas que tradicionalmente se ha asociado con un crecimiento más bajo de la productividad, tanto laboral como multifactor, ha sido el comportamiento del sector servicios ${ }^{1}$, debido al papel central que juega en las economías modernas, tanto cuantitativa como estratégicamente. Una de las cuestiones abiertas sobre este tema es si este crecimiento más bajo de la productividad podría deberse a una menor innovación relativa en el sector servicios².

En la actualidad las economías desarrolladas se han caracterizado, entre otras cosas, por un crecimiento sostenido del peso de los servicios, tanto en el empleo como en la producción. Dos de los principales temas sobre los que los economistas se han preocupado respecto al sector servicios son, por una parte, la productividad y, por otra parte, la innovación en y a través de estas actividades. Estas dos cuestiones están obviamente conectadas, si se acepta que la actuación económica a medio y largo plazo está fuertemente ligada al dinamismo de la innovación. La existencia de este dualismo justifica el presente trabajo. A pesar de que las relaciones entre productividad e innovación han sido ampliamente analizadas en los últimos años, los análisis para el caso del sector servicios no han sido tan exhaustivos hasta el momento. El presente trabajo trata de cubrir este hueco de la literatura existente al profundizar en el análisis del sector servicios y sus ramas de actividad para el caso de las TIC.

La cuestión de la productividad es un puzzle en muchos servicios de los tradicionalmente denominados "en estancamiento", donde los datos muestran bajas, o incluso negativas, tasas de crecimiento; mientras que los estudios de caso y los análisis empíricos más profundos revelan que algunos sectores son de hecho tan o más dinámicos que

1 Aunque dicha tesis fue introducida de forma pionera por Fourastie (1949), no fue hasta los trabajos de W. Baumol y su famosa "enfermedad de costes" (Baumol, 1967) cuando dicha hipótesis sobre la baja productividad de los servicios se generalizó en la literatura especializada. Véase Maroto (2009 y 2010a) para una revisión exhaustiva sobre las relaciones entre productividad y sector servicios en la literatura especializada.

${ }^{2}$ Véase, entre otros, OCDE (2001a, 2005), Rubalcaba (2007), Uppenberg y Strauss (2010), Segarra (2010), o el número especial 32(2) de Abril de la revista Canadian Journal of Economics (1999) sobre productividad en el sector servicios y la paradoja de al productividad, concepto en el que se profundizará en la siguiente sección. 
muchas de las industrias manufactureras ${ }^{3}$. La pregunta que surge es ¿cómo conciliar estas dos posturas contrarias? Multitud de argumentos se han puesto sobre la mesa a lo largo de los años, aunque ninguno acaba de responder completamente a esta pregunta. Por esta razón, no tendría sentido rechazar la hipótesis de la existencia de errores y sesgos en la conceptualización y medición de la productividad en los servicios ${ }^{4}$.

Por su parte, algo similar ocurre cuando el centro del análisis es la innovación en y a través de los servicios. Muchos de los conceptos y teorías existentes indudablemente constituyen útiles herramientas para entender en alguna medida los procesos de innovación en los servicios. Sin embargo, al mismo tiempo, la mayoría de estas aproximaciones tienen un sesgo hacia las manufacturas (Griliches, 1994) y no capturan correctamente algunos aspectos fundamentales de la innovación terciaria (Gallouj, 2002a,b). Igual que tradicionalmente se ha dicho que los niveles de productividad son bajos, la innovación en los servicios a menudo se considera no existente o limitada a la adopción de tecnologías originadas en las manufacturas (Pavitt, 1984).

El principal argumento que interrelaciona los dos conceptos tratados en este trabajo: productividad e innovación (en su vertiente tecnológica a través de las TIC), radica en la siguiente base. Se ha demostrado extensamente que el crecimiento económico a medio y largo plazo depende crucialmente del dinamismo y formas de innovación. Como los servicios contabilizan unos tres cuartos de la actividad económica en los países occidentales, entonces es muy probable que la innovación ligada a los servicios podría ser un factor determinante de la productividad, y, más ampliamente, de la actuación económica a nivel global. Sin embargo, el mayor nexo entre ambas variables se localiza en el ámbito conceptual y puede resumirse en las siguientes palabras clave: co-producción, calidad de servicio, $y$, fundamentalmente, conocimiento, ya que tanto la innovación como la productividad en los servicios surge crecientemente de la movilización del conocimiento y la inteligencia, uno de los pilares de las nuevas tecnologías de la información y la comunicación (TIC).

${ }^{3}$ Ver, entre otros, Bosworth y Triplett (2007) y Triplett y Bosworth (2004) para el caso de los Estados Unidos; Crespi et al. (2006) en el Reino Unido; McLachlan et al. (2002) en Australia; Maroto y Cuadrado (2009) para la OCDE; Maroto y Rubalcaba (2008) para el caso de la Unión Europea, o Cuadrado y Maroto (2006) y Maroto (2009a) para el caso español.

4 Ver, entre otros, Maroto (2009a), Ahmad et al. (2003), Elderidge (1999), Lebow y Ruud (2001) o Wölfl (2003) para más detalles sobre los sesgos y problemas de medición de la productividad en el sector servicios. 
Teniendo en cuenta todo esto, este trabajo estudia las interrelaciones entre la innovación tecnológica en servicios y la productividad. El horizonte temporal abarca desde 1980 en adelante y el ámbito geográfico comprende una muestra de países de la OCDE. El objetivo de este artículo es presentar evidencia empírica sobre el impacto que tiene la innovación tecnológica (TIC) sobre la productividad en el sector servicios para el ámbito geográfico y temporal anteriormente mencionado. En concreto, el trabajo se estructura alrededor de tres preguntas: 1 . ¿Cuál es el papel del capital tecnológico en la evolución de la productividad? 2. ¿Puede explicarse la diferente evolución entre Europa y otros países de la OCDE a través del comportamiento de dicho capital tecnológico? Y, finalmente, 3. ¿Dicho papel y evolución difieren significativamente en el caso del sector servicios? El principal valor añadido de la presente investigación radica en el análisis pormenorizado del sector servicios, desagregando en sus diferentes ramas de ocupación - y no tanto en función del uso o producción de TIC como en los trabajos hasta ahora realizados. Además, se utilizan de forma complementaria dos de las técnicas más utilizadas en la literatura especializada: la contabilidad del crecimiento y las técnicas econométricas.

De acuerdo con estas ideas el trabajo se estructura de la siguiente manera. Tras esta breve introducción, la sección 1 revisa las relaciones entre productividad, crecimiento económico y nuevas tecnologías a lo largo de la literatura económica. Dicha sección sirve de marco de referencia para el análisis empírico aplicado posterior. La sección 2 describe las fuentes estadísticas y aproximaciones metodológicas utilizadas en las dos secciones siguientes. En la sección 3 se realiza un análisis descriptivo de la contribución del capital tecnológico al crecimiento de la productividad utilizando aproximaciones tradicionales basadas en la contabilidad del crecimiento. Mientras que en la sección 4 se presentará un modelo dinámico sobre el papel del capital tecnológico en el crecimiento de la productividad a través de un modelo de datos de panel para algunos países europeos durante el período 1980-2006. El trabajo concluye con algunas consideraciones finales.

\section{RELACIONES ENTRE PRODUCTIVIDAD, CRECIMIENTO Y TECNOLOGÍAS DE LA INFORMACIÓN Y LA COMUNICACIÓN (TIC)}

El objetivo de esta revisión literaria es ofrecer un marco de referencia sobre los argumentos teóricos y resultados empíricos a cerca del impacto económico de las innovaciones en las tecnologías de la información, comúnmente denominadas como Nueva 
Economía ${ }^{5}$. El interés por este fenómeno se acrecentó a mediados de los 90 s a raíz del extraordinario comportamiento de la economía estadounidense en materia de productividad y empleo, relacionado por muchos autores con la posición dominante de EE.UU. en la difusión y uso de las TIC, inversión en I+D o cualificación de la fuerza laboral (Jorgenson, 2001, Jorgenson y Stiroh, 2000). Dicha posición dominante de la economía estadounidense y el diferencial creciente con respecto a los países europeos dio lugar a toda una corriente de pensamiento y análisis empírico que giraba en torno al concepto de la paradoja de la productividad, en el que se profundizará más adelante.

La mayoría del debate y análisis empírico sobre dicho fenómeno utiliza, metodológicamente hablando, los modelos de producción neoclásicos, a partir de los trabajos seminales de Abramovitz (1956) y Solow (1957). En esta línea, algunos autores enfatizaron los problemas de medición como consecuencia del papel cuantitativo de la productividad total de los factores ${ }^{6}$ (PTF) o componente residual (Griliches, 1995a). De forma paralela, otra rama de dicha corriente - dentro de los modelos de crecimiento endógeno - centró su interés en explicar las causas del crecimiento de dicho componente residual. Dentro de esta rama se encuentran, entre otros, aquellos modelos relacionados con la generación de conocimientos (Romer, 1990), la inversión en I+D (Griliches, 1973 y 1979) y sus externalidades (Arrow, 1962; Griliches, 1992 y 1995b; O’Mahony y Vecchi, 2002), o la acumulación de capital humano (Uzawa, 1965; Lucas, 1988). Estas contribuciones, así como otras relacionadas, reconocen la naturaleza endógena y

${ }^{5}$ Aunque no existe un consenso generalizado sobre la definición y concepto de Nueva Economía, generalmente dicho término hace referencia al impacto derivado de la inversión y uso de las TIC - principalmente durante la década de los 90s en los Estados Unidos -. Dicho concepto suele ir asociado a otros como los de Economía del Conocimiento ya que el uso de las TIC precisa en muchos casos de capital humano y conocimiento para que la inversión realizada de sus frutos en términos de productividad. Bajo el paraguas de Nueva Economía se incluyen temas relacionados no sólo con las TIC, sino también con otros campos como la biotecnología, el comercio electrónico e Internet, el incremento en la utilización de mano de obra altamente cualificada o el aumento en el gasto en actividades de I+D (EPKE, 2002).

${ }^{6}$ Dentro del concepto de productividad total de los factores se incluyen generalmente sólo el factor trabajo y el factor capital, excluyendo otra serie de insumos como la tierra, la energía, los servicios intermedios... Por esta razón, muchos autores y manuales (OCDE, 2001b; BLS, 1997) han optado por denominar dicho concepto como productividad multifactorial (PMF), dejando el término de PTF para el caso en el que se incluyen todos los factores productivos en su estimación. 
dinámica del cambio tecnológico y tienen en cuenta alguna de las peculiares caras del proceso de innovación.

Sin embargo, el marco neoclásico no ha sido el único utilizado en la literatura económica en esta área. Las principales críticas a los modelos anteriormente descritos han venido por parte de las teorías evolutivas y eclécticas. Dentro de este amplio conjunto teórico, podemos principalmente distinguir dos corrientes de pensamiento. En primer lugar, aquellos modelos evolutivos basados en la heterogeneidad biológica de los agentes (Soete y Turner, 1984; Nelson y Winter, 1982; Silverberg y Verspagen, 1994). Segundo, las contribuciones sobre crecimiento y cambio tecnológico basadas en metodologías eclécticas (Pérez, 1983, 1985; Freeman y Soete, 1997; Dosi et al., 1990; y Petit y Soete, 2001) donde la generación de conocimiento se analiza dentro de un proceso dinámico de afirmación de un nuevo paradigma tecno-económico (Freeman, 1987; David, 1990, 1991 y 2000).

Veamos ahora el impacto de las TIC sobre la productividad y el crecimiento a través de los principales trabajos empíricos sobre la materia. Uno de los principales focos de atención en esta área es la conocida como paradoja de la productividad ${ }^{7}$. Algunas de las razones para explicar dicha paradoja fueron los problemas de medición (Siegel, 1994; Schreyer, 1998; Brynjolfsson, 1993; Attewell, 1994), problemas de escala en la inversión en TIC (Triplett, 1999) o el posible retardo en la consecución de resultados de dicha inversión (David, 1990; Caselli, 1999). El debate sobre este fenómeno persistió durante los 90 s y parece haber culminado con el consenso de que no existe tal paradoja, debido al papel del capital tecnológico en el fuerte crecimiento de la productividad en EE.UU. a partir de 1995 (Oulton, 2001; Triplett y Bosworth, 2003 y 2005; Bosworth y Triplett, 2007).

En este punto el debate ha virado a si la economía estadounidense está experimentando un milagro de la productividad ${ }^{8}$ derivado de las tecnologías de la información o no. Mientras que la evidencia hasta mitad de los 90 s observaba únicamente un impacto

${ }^{7}$ Dicho concepto nace por el hecho observable de que la economía estadounidense experimentó una caída en el crecimiento de su productividad desde mediados de los 70s hasta mediados de los 90s, que coincidió con sustanciales inversiones en TIC (Strasssman, 1985; Loveman, 1988; Berndt y Morrison, 1992). Algunos trabajos sobre la paradoja de la productividad son, entre otros, los de Roach (1991), Brynjolfsson y Hitt (1996 y 1998); Crafts (2002); Diewert y Fox (1999); Mason et al. (2000); Wolff (1999) o Pilat (2004).

${ }^{8}$ Mientras que la tasa de crecimiento medio anual de la productividad en EE.UU. entre 1974 y 1990 fue del 1.3 por 100; y en los primeros años 90s. del 1.5 por 100; entre 1996 y 2006 ha sido del 2.3 por 100, según los datos ofrecidos por la base EUKLEMS (Maroto y Cuadrado, 2008). 
modesto de las TIC sobre el crecimiento económico (Oliner y Sichel, 1994; Jorgenson y Stiroh, 1995); los últimos análisis han llevado a la afirmación de que las TIC son el principal factor de la aceleración en el crecimiento de la productividad estadounidense (Gordon, 2000 y 2001; CEA, 2000 y 2001; Jorgenson, 2001; Jorgenson y Stiroh, 2000; Oliner y Sichel, 2000 y 2002; Whelan, 2000), con lo que incluso se ha declarado que la paradoja de la productividad, originalmente introducida por Solow en 1987, ha quedado obsoleta. Todos estos trabajos concluyen que ha habido un importante efecto de capitalización, derivado del aumento en la inversión en capital relacionado con las TIC, y que se ha incrementado la PTF en aquellos sectores productores de TIC. El hecho de que cada trabajo se focalice en diferentes horizontes temporales y sectores económicos hace que el impacto de las TIC sobre el crecimiento de la productividad varíe sensiblemente. En general, todos coinciden en contabilizar alrededor de un tercio del crecimiento de la productividad laboral en los EE.UU. por el efecto de una mayor capitalización a partir de 1995 con respecto al período anterior. De este efecto, casi la totalidad (aproximadamente un 40 por 100) se debe a inversiones en capital TIC.

Aunque todas estas evidencias de la economía estadounidense parecen mostrar un claro impacto de las TIC en el crecimiento agregado, todavía quedaría por resolver la cuestión sobre si este efecto es temporal o afecta al crecimiento a largo plazo. Autores como Jorgenson (2001), Gordon (2000) o David (2000) concluyen que la actual revolución de las TIC es únicamente uno de los muchos cambios que permitirán un crecimiento sostenido a largo plazo. Sin embargo, la mayoría sugieren que todavía es pronto para predecir la sostenibilidad a largo plazo de este crecimiento de la productividad estadounidense (Stiroh, 1999; De Masi, 2000).

Hasta ahora, esta revisión bibliográfica se ha centrado en el caso estadounidense, por su comportamiento positivo en los últimos años. Sin embargo, un amplio conjunto de trabajos recientes han tratado el impacto de las TIC sobre el crecimiento y la productividad en otras áreas económicas desarrolladas. Muchos de ellos consideran países individuales ${ }^{9}$ y los comparan con EE.UU. Otros autores consideran grupos de países, como Schreyer (2000) para el G-7, Colecchia y Schreyer (2001) para un conjunto de nueve países OCDE, o Vijselaar y Albers (2002) para el área Euro. Uno de los países en

${ }^{9}$ Entre otros, Armstrong et al. (2002) presentan el caso de Canadá; Oulton (2001) el del Reino Unido; Cette et al. (2002) el de Francia; y van der Wiel (2001) el de Holanda. Crafts y O'Mahony (2001) comparan los niveles de productividad de EE.UU., Francia, Alemania y el Reino Unido. 
los que más han proliferado este tipo de estudios es, precisamente, España ${ }^{10}$. La razón fundamental ha sido el paradójico comportamiento que ha experimentado nuestra productividad en los últimos años (Maroto y Cuadrado, 2008; Maroto, 2009b), ya que las dinámicas tasas de crecimiento económico y creación de empleo no se han traducido en una positiva evolución de la productividad, tanto laboral como multifactorial.

Una conclusión común en todos los trabajos anteriormente citados es que existen notables diferencias en el impacto de las TIC sobre la productividad según el sector económico que se analice. Por esta razón, un amplio conjunto de la literatura más reciente ha analizado estas relaciones a nivel sectorial. La mayoría de estos trabajos distinguen los sectores según su grado de utilización de las TIC, como los de Stiroh (2001), Baily y Lawrence (2001), O’Mahony y de Boer (2002), Mas y Quesada (2006), van Ark (2001), Inklaar et al. (2005), van Ark et al. (2003) o van Ark e Inklaar (2007). Todos estos autores concluyen que tanto los sectores productores como usuarios de TIC son importantes a la hora de explicar el crecimiento de la productividad estadounidense a partir de 1995; mientras que el retraso relativo en los países europeos se debe, fundamentalmente, al peor comportamiento en los sectores donde el uso del capital TIC es menos intensivo. Sin embargo, no toda la evidencia sectorial existente es favorable a dicho impacto de las TIC sobre el crecimiento ${ }^{11}$. El informe del McKinsey Global Institute (2001), por ejemplo, matiza dicha afirmación, diciendo que la inversión en TIC es únicamente uno de los muchos factores a la hora de explicar el mejor comportamiento en materia de productividad de EE.UU. con respecto a Europa. Además, los análisis específicamente econométricos tampoco encuentran evidencia del impacto de aquellos sectores intensivos en TIC (Stiroh, 2002; O’Mahony y de Boer, 2002).

A pesar del amplio abánico de trabajos sobre las relaciones entre TIC y productividad, tanto a nivel agregado como sectorial, son todavía pocos los trabajos que han profundizado en el caso del sector servicios en particular. Todos los trabajos anteriormente mencionados distinguen en función del uso o producción de TIC y no de las ocupaciones laborales. En concreto, únicamente van Ark et al. (2002) analizan el sector servicios en profundidad aunque tampoco lo hagan distinguiendo las distintas ramas de ocupación

${ }^{10}$ Ver entre otros Mas y Robledo (2010), Billón et al. (2007); Mas y Quesada (2005); Gómez et al. (2006); Hernansanz et al. (2001); Núñez (2001); Hernando y Nuñez (2002) o Pérez et al. (2006).

${ }^{11}$ Sin embargo, estos resultados aparentemente negativos deben tratarse con cautela ya que dependen en gran medida de cómo se construya la función de producción y cómo se estime el componente residual de la misma. 
terciaria. En cualquier caso, la importancia de la paradoja de la productividad dista de ser homogénea para todas las ramas de servicios, asociándose este fenómeno, en gran medida, a las mayores o menores dificultades en la medición del output de las ramas de servicios. Es por esta dificultad, que existen pocos trabajos que aborden comparaciones a nivel internacional de niveles y crecimiento de la productividad sectorial.

En conclusión, como se ha podido observar, han sido muchos los autores que han tratado el impacto de la llamada Nueva Economía en general, y las TIC en particular, en el crecimiento y la productividad en los últimos años. El primer resultado que puede deducirse de estos trabajos es que hay amplia evidencia a nivel agregado de que las TIC han incrementado la productividad laboral a través de una mayor capitalización relacionada con estas nuevas tecnologías. Igualmente, la PTF se ha incrementado en aquellos sectores productores de nuevas tecnologías; mientras que la evidencia no es tan concluyente para aquellas actividades usuarias de TIC. Finalmente, también hay que tener en cuenta el impacto del incremento en la demanda de mano de obra cualificada, las inversiones intangibles como las de I+D y los cambios organizacionales derivados de la implantación de las TIC, en el crecimiento de la productividad, tanto laboral como multifactorial. El objetivo de las siguientes secciones será analizar si estas conclusiones pueden aplicarse al caso del sector servicios y, si es así, si a todas las ramas de servicios por igual.

\section{DATOS Y METODOLOGÍA}

Para explorar las relaciones existentes entre el papel de los principales sectores de actividad (entre ellos el sector servicios) y la evolución de la productividad en los últimos años hemos elegido trabajar con la base EUKLEMS. Esta fuente, cuya elaboración fue el principal objetivo de un proyecto internacional para la Comisión Europea, ofrece exhaustiva información sobre crecimiento económico, productividad, creación de empleo, formación de capital y cambio tecnológico a nivel sectorial (cubriendo más de 70 ramas de actividad) para los países miembros de la Unión Europea, Australia, Corea, Japón, Canadá y Estados Unidos, desde 1970 en adelante. Esta fuente, además, es comparable con otras bases ofrecidas por los principales organismos internacionales, como la OCDE o la Comisión Europea, aunque se ponen de manifiesto ciertas diferencias en las magnitudes estimadas ${ }^{12}$.

12 Véase Timmer et al. (2008) para más información sobre dicha base o www.euklems.net 
Desde el punto de vista metodológico, en la sección 3 se analiza la contribución del capital TIC al crecimiento económico y crecimiento de la productividad a través de la contabilidad del crecimiento (Solow, 1957; Jorgenson et al., 1987 y 2005; Jorgenson y Stiroh, 1995). El horizonte temporal es 1980-2006 y el geográfico es una muestra de diez países ${ }^{13}$ de la Unión Europea - por disponibilidad de datos. Según dicho modelo de crecimiento, el valor añadido bruto $(Y)$ se origina a partir de una serie de factores productivos, concretamente capital TIC $\left(K_{I C T}\right)$, capital no TIC $(K)$ y factor trabajo $(L)$. La productividad total de los factores (A) se representa como el progreso técnico Hicks-neutral, con lo que la función de producción tendrá la siguiente forma:

$$
Y=A\left(L, K, K_{I C T}\right)
$$

Bajo los supuestos de productores minimizadores de costes, mercados de factores competitivos y rendimientos constantes a escala, el crecimiento del valor añadido ${ }^{14}$ sectorial puede descomponerse en la contribución del factor trabajo, el capital (tanto tecnológico como no) y la productividad multifactorial:

$$
\Delta \ln Y_{i t}=\bar{w}_{i t}^{L} \Delta \ln L_{i t}+\bar{w}_{i t}^{K} \Delta \ln K_{i t}+\bar{w}_{i t}^{I C T} \Delta \ln K_{i t}^{I C T}+\Delta \ln A_{i t}(1)
$$

donde $\Delta$ expresa primeras diferencias y $\bar{w}$ identifica la media entre dos períodos de tiempo del peso del factor correspondiente sobre el total de renta ${ }^{15}$. Cada sumando de la ecuación (1) representa la contribución de cada factor productivo y de la productividad multifactorial al crecimiento económico, que son las variables que se analizan en detalle en la sección 3 de este trabajo. Además, la contribución del factor trabajo se ha descompuesto en la contribución de las horas trabajadas $\left(\bar{w}_{i t}^{L} \Delta \ln H_{i t}\right)$ y un efecto de la composición de la mano de obra ${ }^{16}\left[\bar{w}_{i t}^{L}\left(\Delta \ln L_{i t}-\Delta \ln H_{i t}\right)\right]$.

${ }^{13}$ Por disponibilidad de datos homogéneos y completos, se trabajará con UE-10, compuesta por Bélgica, Austria, Dinamarca, Finlandia, Francia, Alemania, Italia, España, Suecia y Reino Unido.

${ }^{14}$ Se utiliza el valor añadido, en lugar del producto bruto, debido a que dicho indicador es mejor para los análisis sectoriales, como el que nos ocupa.

15 Como se ha supuesto rendimientos constantes a escala, entonces $\bar{w}_{L}+\bar{w}_{K}+\bar{w}_{I C T}=1$.

${ }^{16}$ En función de tres características de la mano de obra: género, edad y nivel de educación. 
Despejando de la ecuación (1), y llamando $y=Y / L$ al crecimiento de la productividad aparente del trabajo, se puede descomponer dicho crecimiento en la contribución del ratio capital-trabajo (tanto para el capital tecnológico $k_{I C T}$ como no tecnológico $k$ ) y la contribución de la productividad multifactorial, según la ecuación (2):

$$
\Delta \ln y=\bar{v}_{K} \Delta \ln k+\bar{v}_{I C T} \Delta \ln k_{I C T}+\Delta \ln a
$$

Como se adelantó en la introducción, el presente trabajo combina dos de las técnicas más extendidas en el análisis de las relaciones entre productividad e innovación. Mientras que en la sección 3 se utilizan técnicas de contabilidad de crecimiento, en la sección 4 se aplican técnicas econométricas. De esta forma, la sección 4 aborda el papel que ostenta la capacidad innovadora, al menos tecnológica, de los servicios a nivel macroeconómico a través de la contribución del capital de TIC, fuente indiscutible de innovación en servicios, al crecimiento y la productividad del sector. Para ello, se utiliza un panel de datos con los mismos diez países europeos que se utilizaba en la sección anterior, más Estados Unidos, y el mismo horizonte temporal.

Siguiendo la notación tradicional anteriormente introducida en el marco metodológico para la sección 3, se parte de una función de producción (de tipo Cobb-Douglas) que relaciona el valor añadido con los inputs que se utilizan para conseguirlo. En forma logarítmica y tomando crecimientos, se estima un modelo de efectos aleatorios ${ }^{17}$ que relaciona el crecimiento del valor añadido con la contribución de los tres inputs considerados (trabajo, capital no tecnológico y capital TIC):

$$
y_{i t}=\alpha_{i}+\gamma_{1} l_{i t}+\gamma_{2} k_{i t}+\gamma_{3} k_{i t}^{I C T}+\varepsilon_{i t}
$$

donde $y, l, k$ e $k_{I C T}$ son las tasas de crecimiento anual, respectivamente, del valor añadido bruto $(Y)$, servicios de trabajo $(L)$, servicios de capital no TIC $(K)$ y servicios de capital TIC (o ICT, de las siglas en inglés). Los parámetros $\gamma_{i}$ estiman el peso de

17 Se ha escogido el modelo de efectos aleatorios siguiendo los resultados del test de Hausman (que aceptan la hipótesis de que las diferencias entre los coeficientes de efectos aleatorios y fijos no son significativos) y del test de Breusch y Pagan (que rechazan la hipótesis de que los efectos aleatorios no sean significativos). 
cada factor en el valor añadido nominal. Los subíndices se refieren al país $(i=1, . ., 11)$ y año $(t=1, \ldots, 26)$, mientras que la variable $\alpha_{i}$ estima el efecto fijo para cada país de la muestra escogida.

A partir de la ecuación (3), el siguiente paso ha sido estimar la contribución de cada factor productivo sobre el crecimiento de la productividad aparente del trabajo, obteniéndose la siguiente relación en términos logarítmicos:

$$
l p_{i t}=\left(\frac{y}{l}\right)_{i t}=\tilde{\alpha}_{i}+\beta_{1}\left(\frac{k}{l}\right)_{i t}+\beta_{2}\left(\frac{k_{I C T}}{l}\right)_{i t}+\xi_{i t}
$$

A continuación se aplica esta metodología a la base de datos especificada para analizar el efecto o papel del capital TIC y la innovación tecnológica en el crecimiento de la productividad, poniendo especial atención a lo que sucede en el caso del sector servicios, así como las heterogéneas ramas de actividad que lo componen, y tomando como referencia los países con datos disponibles de la Unión Europea, entre ellos la economía española.

\section{CONTRIBUCIÓN DEL CAPITAL TECNOLÓGICO AL CRECIMIENTO DE LA PRODUCTIVIDAD. APROXIMACIÓN A TRAVÉS DE LA CONTABILIDAD DEL CRECIMIENTO}

Tradicionalmente, dos de los tópicos con los que se ha caracterizado el sector servicios han sido el de su bajo crecimiento de la productividad y el de su bajo nivel de innovación. Si esto fuera así, la transición hacía economías de servicios, como las que caracterizan las sociedades modernas, llevaría a un estancamiento en el crecimiento y menores tasas de desarrollo tecnológico (Gadrey y Gallouj, 2002). Sin embargo, la evidencia empírica parece matizar o limitar este tipo de "mitos" sobre los servicios. Los servicios se muestran cada vez más como una parte dinámica de las economías. Como se verá, muchas de las actividades de servicios muestran altas tasas de crecimiento de la productividad, son altamente innovadoras y sus puestos de trabajo requieren una mano de obra altamente cualificada. Igualmente, los servicios cada día están más internacionalizados y se enfrentan a una mayor competencia, y la inversión en TIC, promotora de avances productivos, cada día juega un papel más importante (Pilat, 2001). 
FIGURA 1.-Relación entre innovación (SII = indicador sintético de innovación, 2003) y productividad por trabajador, 1980-2006

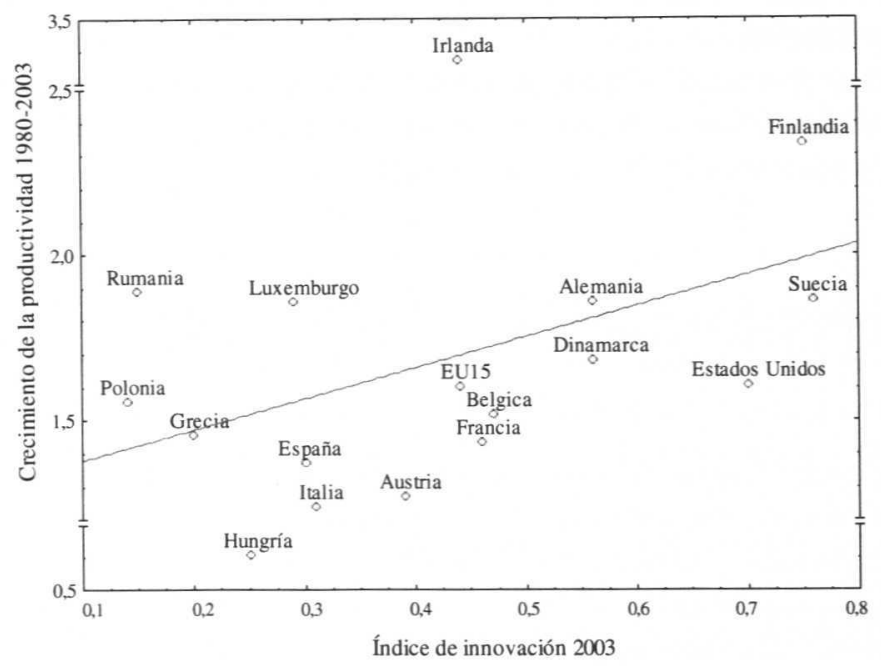

Fuente: Elaboración propia a partir de datos de Eurostat y EUKLEMS.

La Figura 2 ofrece una primera imagen que relaciona las dos variables objeto de estudio de este apartado: la productividad y la innovación. En dicho gráfico se puede observar como parece existir una relación entre el crecimiento de la productividad y el nivel de innovación en la muestra de países escogida. Aquellos países con un mayor índice de innovación (Finlandia, Suecia y Estados Unidos), son también los que alcanzan mayores crecimientos medios de su productividad desde 1980 en adelante.

A continuación se presentará evidencia empírica internacional sobre la influencia del capital tecnológico o capital TIC en el crecimiento de la producción y la productividad en el sector servicios. Para ello se utiliza una aproximación clásica, basada en la contabilidad del crecimiento, como se ha descrito en la sección segunda.

La evidencia empírica indica que durante la segunda mitad de los 90 el patrón de crecimiento en la Unión Europea ha experimentado un cambio significativo. Aunque se han mantenido unas tasas de crecimiento económico similares a las de la etapa anterior, no ha ocurrido lo mismo con las tasas de crecimiento de la pro- 
ductividad, que se han reducido notablemente. Esta ralentización estructural en la productividad europea como conjunto es aún más preocupante cuando se compara con la evolución de Estados Unidos, cuya productividad se ha acelerado significativamente en el mismo período de tiempo.

Respecto a la contribución sectorial al crecimiento agregado, el sector manufacturero y, especialmente, aquellos sectores productores de TIC, han contribuido significativamente al crecimiento en Europa. Sin embargo, las diferencias con respecto a Estados Unidos en materia de productividad se deben, principalmente, a la menor contribución de los servicios de mercado, en especial aquellos de distribución, así como los financieros y los servicios a empresas (Bosworth y Triplett, 2007; Schreyer, 2000; Maroto, 2010b). En términos de las fuentes del crecimiento económico, la contribución del factor trabajo en Europa ha aumentado a partir de 1995 (dos tercios de este aumento debidos al crecimiento en el número de horas trabajadas, mientras que el resto se debería a una mejora en la composición de la mano de obra). La contribución del factor capital no ha cambiado mucho, en términos agregados, aunque sí se puede observar un desplazamiento de la distribución a favor de aquellos servicios de capital TIC. Sin embargo, este cambio hacia un uso más intensivo del capital TIC no ha sido tan pronunciado como en la economía estadounidense (salvo en algunos países concretos, como Holanda o Reino Unido). Finalmente, el principal factor de la caída en el crecimiento de la productividad en Europa ha sido la caída en el crecimiento de la productividad multifactorial. Este fenómeno ha sido de especial importancia en países como Italia o España, donde las tasas de crecimiento han sido incluso negativas, pudiendo estar reflejando una falta de aprovechamiento de la economía del conocimiento, de los sistemas y externalidades de la innovación, y, en su caso, rigideces en el funcionamiento de los mercados (Timmer et al., 2008).

Las Tablas 1 y 2 resumen la evolución de la contribución de cada factor al crecimiento del valor añadido a nivel sectorial, distinguiendo entre el período 19801995 y la etapa posterior a 1995, para la muestra de UE-10. Se distingue entre el total de la economía y los dos principales sectores: manufacturas y servicios, desagregando éstos en comunicaciones ${ }^{18}$, servicios de mercado (sin comunicaciones) y servicios de no mercado. Los servicios de mercado incluyen los servicios

${ }^{18}$ Los servicios de comunicación se separan del resto de servicios dado el peculiar comportamiento en productividad observado en anteriores trabajos, como el de O'Mahony y Van Ark (2003) o Maroto (2010b). 
de comercio (50-52), hoteles y restaurantes (55), transportes (60-63), finanzas y seguros (55-57), servicios a empresas (71-74) y otros servicios (90-93). Mientras que los servicios de no mercado integran los servicios públicos (75), educación (80), sanidad (85) y actividades inmobiliarias (70).

Se puede observar que el crecimiento del valor añadido en los servicios ha sido superior al de las manufacturas, especialmente a partir de mediados de los 90. Otro hecho distintivo es la contribución del factor trabajo. Mientras que en las manufacturas tiene una contribución claramente negativa, debido fundamentalmente al descenso en el número de horas trabajadas, en los servicios contribuye de modo muy significativo, especialmente en el segundo periodo. En cuanto a la contribución del capital, ésta es superior en las manufacturas, aunque el porcentaje de la misma que puede atribuirse al capital TIC es mayor en el sector servicios respecto al peso del capital no TIC. La contribución del capital TIC de los dos grandes grupos de servicios aumenta del primer al segundo periodo. Sin embargo, este proceso de capitalización TIC de los servicios no parece haberse reflejado en una mejora de su productividad. Se puede observar que la contribución de la productividad multifactorial al crecimiento del valor añadido no sólo ha caído en los servicios a partir de mediados de los 90 , sino que incluso es negativa, lo que contrasta con el positivo comportamiento de algunos sectores como las telecomunicaciones. 
TABLA 1.-Contribución factorial al crecimiento del valor añadido. Unión Europea-10, 1980-1995 (tasa de crecimiento media anual, en \%)

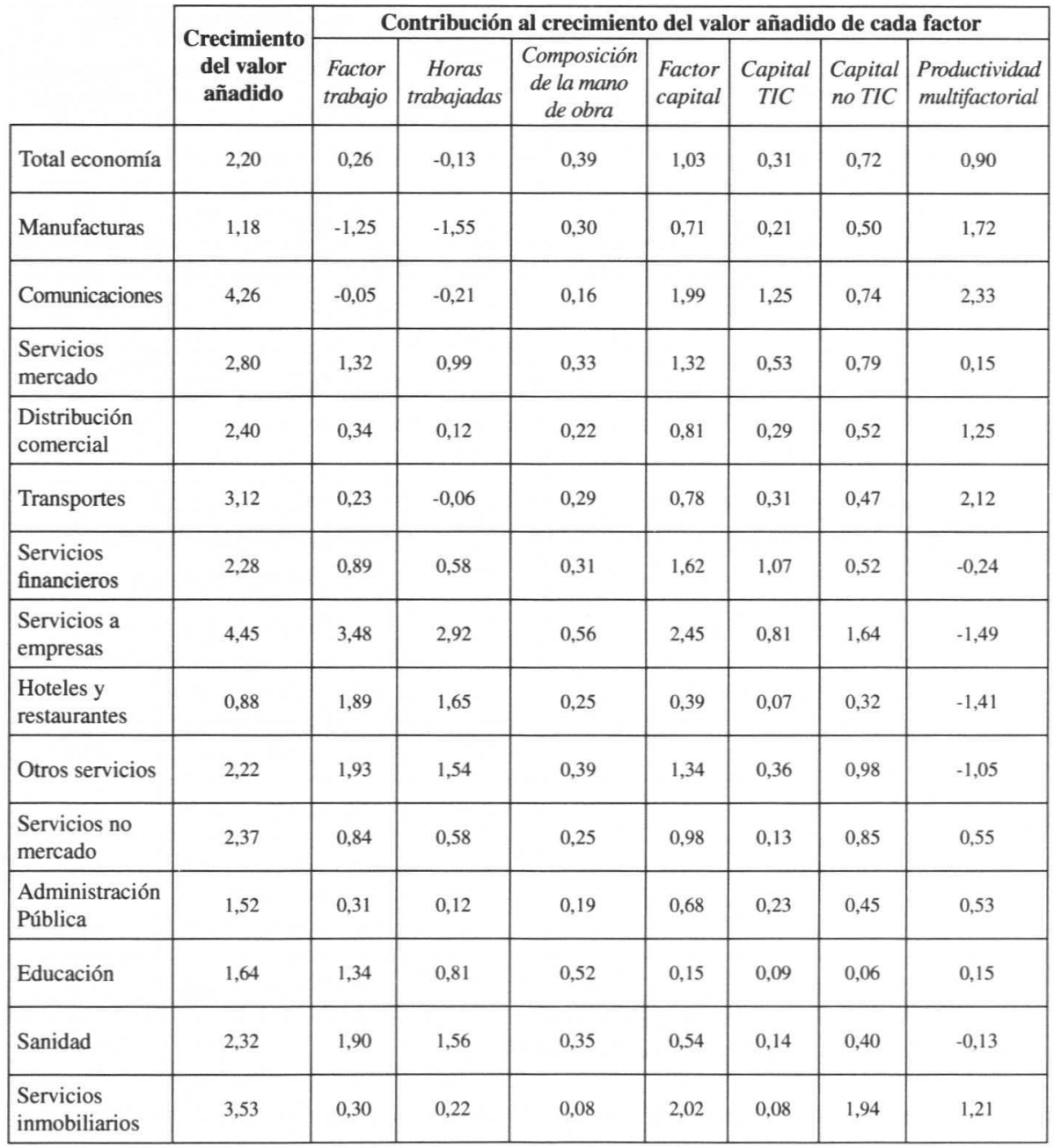

Fuente: Elaboración propia con datos EUKLEMS 
TABLA 2.-Contribución factorial al crecimiento del valor añadido. Unión Europea-10, 19962006 (tasa de crecimiento media anual, en \%)

\begin{tabular}{|l|c|c|c|c|c|c|c|c|}
\cline { 3 - 8 } \multicolumn{1}{c|}{} & \multirow{2}{*}{$\begin{array}{c}\text { Crecimiento } \\
\text { del valor } \\
\text { añadido }\end{array}$} & $\begin{array}{c}\text { Factor } \\
\text { trabajo }\end{array}$ & $\begin{array}{c}\text { Horas } \\
\text { trabajadas }\end{array}$ & $\begin{array}{c}\text { Composición } \\
\text { de la mano } \\
\text { de obra }\end{array}$ & $\begin{array}{c}\text { Factor } \\
\text { capital }\end{array}$ & $\begin{array}{c}\text { Capital } \\
\text { TIC }\end{array}$ & $\begin{array}{c}\text { Capital } \\
\text { no TIC }\end{array}$ & $\begin{array}{c}\text { Productividad } \\
\text { multifactorial }\end{array}$ \\
\hline Total economía & 2,04 & 0,68 & 0,52 & 0,16 & 1,14 & 0,45 & 0,69 & 0,22 \\
\hline Manufacturas & 0,90 & $-0,45$ & $-0,71$ & 0,26 & 0,59 & 0,26 & 0,33 & 0,76 \\
\hline Comunicaciones & 7,64 & 0,13 & $-0,10$ & 0,23 & 2,39 & 1,73 & 0,66 & 5,12 \\
\hline $\begin{array}{l}\text { Servicios } \\
\text { mercado }\end{array}$ & 2,74 & 1,43 & 1,23 & 0,20 & 1,53 & 0,75 & 0,78 & $-0,23$ \\
\hline $\begin{array}{l}\text { Distribución } \\
\text { comercial }\end{array}$ & 2,17 & 0,50 & 0,39 & 0,11 & 1,08 & 0,44 & 0,64 & 0,59 \\
\hline Transportes & 2,73 & 0,93 & 0,76 & 0,17 & 1,28 & 0,48 & 0,80 & 0,52 \\
\hline $\begin{array}{l}\text { Servicios } \\
\text { financieros }\end{array}$ & 2,87 & 0,32 & 0,03 & 0,29 & 1,40 & 1,22 & 0,18 & 1,15 \\
\hline $\begin{array}{l}\text { Servicios a } \\
\text { empresas }\end{array}$ & 3,93 & 3,10 & 2,80 & 0,30 & 2,53 & 1,24 & 1,29 & $-1,70$ \\
\hline $\begin{array}{l}\text { Hoteles y } \\
\text { restaurantes }\end{array}$ & 1,65 & 1,91 & 1,69 & 0,22 & 0,79 & 0,14 & 0,65 & $-1,05$ \\
\hline Otros servicios & 1,70 & 1,41 & 1,37 & 0,04 & 1,21 & 0,43 & 0,78 & $-0,93$ \\
\hline $\begin{array}{l}\text { Servicios no } \\
\text { mercado }\end{array}$ & 1,61 & 0,77 & 0,57 & 0,20 & 1,03 & 0,20 & 0,83 & $-0,20$ \\
\hline $\begin{array}{l}\text { Administración } \\
\text { Pública }\end{array}$ & 0,94 & 0,01 & $-0,28$ & 0,29 & 0,55 & 0,32 & 0,23 & 0,38 \\
\hline Educación & 0,86 & 1,69 & 1,38 & 0,31 & 0,25 & 0,16 & 0,09 & $-1,08$ \\
\hline Sanidad & 2,55 & 1,72 & 1,45 & 0,26 & 0,70 & 0,23 & 0,47 & 0,13 \\
\hline $\begin{array}{l}\text { Servicios } \\
\text { inmobiliarios }\end{array}$ & 1,94 & 0,21 & 0,16 & 0,05 & 2,00 & 0,14 & 1,86 & $-0,26$ \\
\hline
\end{tabular}

Fuente: Elaboración propia con datos EUKLEMS

A pesar de esta imagen del sector servicios, es necesario un análisis más desagregado del sector debido a la gran heterogeneidad que lo caracteriza. Así, como se ha avanzado ya el mayor crecimiento en el valor añadido en los servicios europeos se ha dado en el sector de las comunicaciones, fundamentalmente a partir de mediados de los $90 \mathrm{~s}$. En este sector, además, se observa una gran aportación tanto del capital TIC como de la productividad multifactorial. En cuanto a los servicios de mercado, hasta 1995 únicamente la distribución comercial y los transportes 
presentan tasas de crecimiento de la productividad multifactorial positivas; tasas que disminuyen claramente a partir de esa fecha, cuando se observa, sin embargo, un positivo crecimiento en los servicios financieros. Este tipo de servicios, además, son los únicos en los que se observa que el capital relacionado con las TIC supera claramente en contribución al resto de capital durante todo el período analizado. En cuanto a los servicios de no mercado, se observa como las tasas de crecimiento de la productividad caen a partir de mitad de los 90 s en todas las actividades analizadas, y que la contribución del capital tecnológico queda lejos de la del resto de capital, con la excepción de los servicios de las Administraciones Públicas.

Por otra parte, además del efecto que pueda tener el capital TIC sobre el crecimiento de la producción, también puede ser interesante contrastar el que pueda tener sobre la productividad aparente del trabajo. Las Figuras 3 y 4 muestran los resultados de dicha descomposición para la Unión Europea-10 en el periodo que va de 1980 a 2006, para los grandes sectores y la desagregación del sector servicios, respectivamente. En cada uno de los sectores tratados las contribuciones de los diferentes factores es realmente diversa. 
FIGURA 2.-Contribución factorial al crecimiento de la productividad laboral. Unión Europea-10

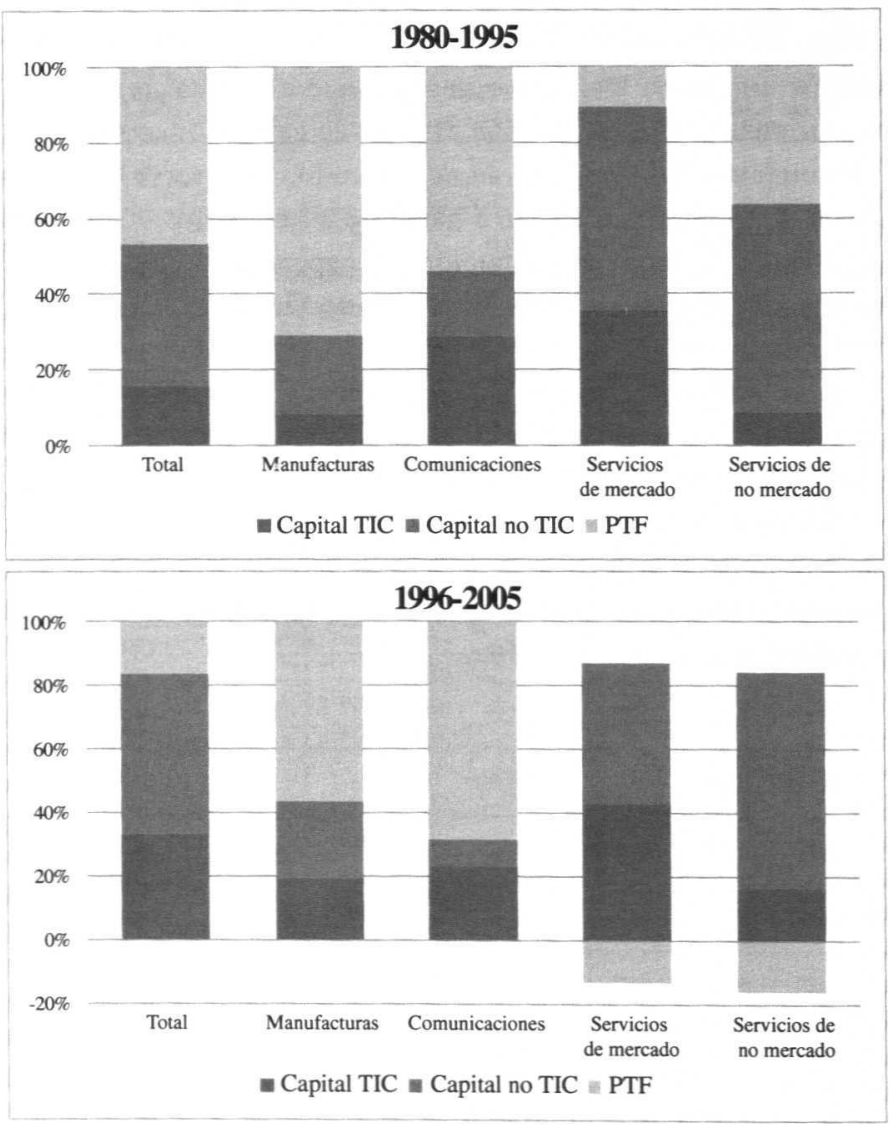

Fuente: Elaboración propia con datos EUKLEMS

A nivel agregado, el primer hecho destacable es el incremento de la contribución del capital TIC al crecimiento de la productividad laboral a partir de 1995 (pasa de un 16 por 100 a un 33 por 100), fundamentalmente gracias a la caída observada por la productividad multifactorial (pasa de un 47 por 100 a un 16 por 100) a partir de esa fecha en los países europeos. Este fenómeno se observa tanto en las manufacturas como en los servicios, donde incluso la contribución de la productividad multifactorial a la productividad laboral a partir de mediados de los 90 s es 
negativa, como se observa en los gráficos de la Figura 3. Únicamente el sector de las comunicaciones se escapa de esta tendencia, ya que su PTF pasa de contribuir en un 54 por 100 al crecimiento de la productividad laboral hasta 1995 a un 68 por 100 a partir de entonces. El segundo hecho destacable es que el capital TIC aparece como un factor relativamente más importante en los servicios de mercado (mucho más que en las manufacturas), gracias, fundamentalmente, a la casi nula aportación de la PMF.

Los datos analizados a partir de los dos periodos expuestos anteriormente (19801995 y 1996-2006) muestran que, en el sector servicios, la contribución del capital TIC al crecimiento de la productividad laboral ha crecido notablemente a partir de 1995, tanto en los servicios de mercado (donde alcanza un 58 por 100 en el período 1996-2006) como en los de no mercado (donde alcanza un $24 \%$ para el mismo período). Sin embargo, como sucedía en el caso del crecimiento de la producción, este aumento de la contribución del capital TIC no se ha traducido en términos de productividad. La contribución de la productividad multifactorial, con la excepción de las comunicaciones, no sólo ha disminuido con respecto a los años anteriores a 1995, sino que es negativa para el período 1996-2006. Estos datos, comparando con la situación en Estados Unidos, sugieren que puede haber un retraso en los impactos de las TIC respecto a la productividad: allí ya habría registrado estadísticamente sus beneficios, mientras que en Europa, donde la incorporación de las TIC ha sido, en general, más tardía, los impactos estarían todavía en fase de consolidación, aunque los datos entre 2004 y 2006 permiten ya ver sus signos.

Respecto a la evidencia en las distintas ramas terciarias, el primer resultado es, nuevamente, la gran heterogeneidad que se observa dentro del sector, lo que hace prácticamente imposible poder sacar conclusiones generales. El negativo comportamiento de la PTF en el crecimiento de la productividad laboral de los servicios a partir de 1995 se observa en actividades como los servicios a empresas, otros servicios (aunque en estos dos casos, dicho comportamiento negativo ya venía del período anterior) y los servicios inmobiliarios. Otras ramas, como el comercio, transportes, hoteles y restaurantes, y AA.PP., también experimentan una caída en la contribución de la PTF, aunque esta siga siendo todavía positiva. Sin embargo, la contribución de la productividad multifactorial al crecimiento de la productividad laboral en servicios como la educación y, especialmente, la sanidad y los servicios financieros ha aumentado notablemente desde 1995 hasta ahora. 
FIGURA 3.-Contribución factorial al crecimiento de la productividad laboral. Unión Europea-10 Sector servicios
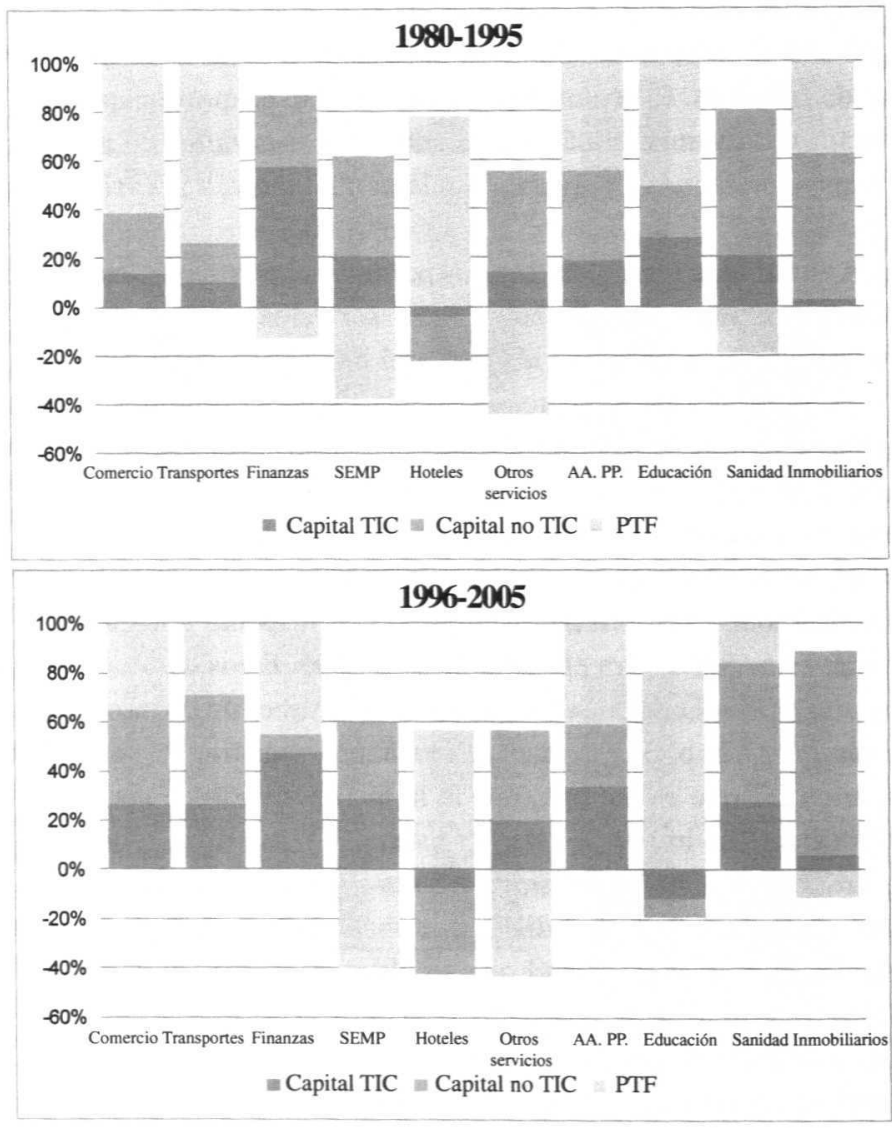

Fuente: Elaboración propia con datos EUKLEMS.

En segundo lugar, el crecimiento del papel del capital TIC en los últimos años que se observa en los servicios a nivel agregado, también se observa cuando se analizan las diferentes ramas de servicios. Este aumento del papel del capital tecnológico es claramente destacado en actividades como el comercio y los transportes, los servicios a empresas, las AA.PP. o la sanidad. Sin embargo, los servicios que mayor contribución reciben del capital TIC son los servicios financieros, donde este tipo de capital ha supuesto dos terceras 
partes del crecimiento de su productividad desde 1980 hasta nuestros días. Sin embargo, otros servicios, fundamentalmente los relacionados con la educación y la hostelería tienen una contribución del capital TIC no sólo relativamente baja, sino incluso negativa durante gran parte del período analizado.

\section{CONTRIBUCión DEl CAPITAL TECNOLÓGiCo AL CRECIMIENTO DE LA PRODUCTIVIDAD. UN MODELO DE DATOS DE PANEL}

Los datos de la sección anterior muestran como el capital relacionado con las nuevas tecnologías ha jugado un papel importante en el crecimiento de la productividad en los países europeos, incluso en las actividades de servicios, tradicionalmente consideradas como poco intensivas en este tipo de capital. Por esta razón, en esta sección se trata de abordar el papel que ostenta la capacidad innovadora, al menos tecnológica, de los servicios a nivel macroeconómico a través de la contribución del capital relacionado con las TIC, fuente indiscutible de innovación en servicios, al crecimiento y la productividad del sector.

Para ello, se implementa un modelo de efectos aleatorios para once países de la OCDE (detallado en la sección 2), estimando modelos de funciones extendidas de producción. Adicionalmente, se comparan estos resultados con los obtenidos mediante técnicas de contabilidad de crecimiento a nivel macroeconómico en la sección anterior. El horizonte temporal de la muestra analizada es 1980-2006. Los resultados de la comparación entre el agregado de la economía, el sector manufacturero y el sector servicios (distinguiendo entre comunicaciones, servicios de mercado y no mercado) se resumen en la Tabla 3.

En primer lugar, puede observarse como los modelos estimados tienen una alta significatividad conjunta en la mayoría de los sectores analizados, ya que presentan un $\mathrm{R}^{2}$ por encima de 0.70 (con la única excepción del modelo para el crecimiento de la productividad en los servicios fuera del mercado, que por otra parte son aquellas actividades de servicios donde la medición y estimación de la productividad presenta mayores dificultades ${ }^{19}$ ). Segundo, el coeficiente para el capital TIC es en todos los casos estadísticamente significativo y positivo, luego parece contrastar los efectos que tiene este tipo de capital en el crecimiento, tanto de la producción como de la productividad,

19 Véase, entre otros, Griliches (1992), Ahmad et al. (2003), Cuadrado (2004), Maroto (2008), Cuadrado y Maroto (2006) y Maroto y Rubalcaba (2008) 
y que se han venido resaltando durante este trabajo. Sin embargo, como se observaba en la evidencia de la sección anterior, todavía está lejos de la contribución del factor trabajo y del resto del capital no tecnológico, como muestran los resultados de la tabla anterior.

TABLA 3.-Relación entre crecimiento económico, productividad y capital TIC. 1980-2006

\begin{tabular}{|c|c|c|c|c|c|}
\hline & \multirow[b]{2}{*}{ Total } & \multirow[b]{2}{*}{ Manufacturas } & \multicolumn{3}{|c|}{ Servicios } \\
\hline & & & Comunicaciones & $\begin{array}{c}\text { Servicios de } \\
\text { mercado }\end{array}$ & $\begin{array}{c}\text { Servicios de no } \\
\text { mercado }\end{array}$ \\
\hline & \multicolumn{5}{|c|}{ Crecimiento del valor añadido ${ }^{1}$} \\
\hline Trabajo $(\gamma 1)$ & $\begin{array}{l}0.3603 \\
(0.000)\end{array}$ & $\begin{array}{l}0.2823 \\
(0.000)\end{array}$ & $\begin{array}{l}0.0018 \\
(0.001)\end{array}$ & $\begin{array}{l}0.4968 \\
(0.000)\end{array}$ & $\begin{array}{l}0.4150 \\
(0.000) \\
\end{array}$ \\
\hline Capital $(\gamma 2)$ & $\begin{array}{l}0.3912 \\
(0.000) \\
\end{array}$ & $\begin{array}{l}0.2975 \\
(0.000) \\
\end{array}$ & $\begin{array}{l}0.7805 \\
(0.000)\end{array}$ & $\begin{array}{l}0.0970 \\
(0.000)\end{array}$ & $\begin{array}{l}0.3066 \\
(0.000) \\
\end{array}$ \\
\hline$T I C(\gamma 3)$ & $\begin{array}{l}0.0814 \\
(0.000) \\
\end{array}$ & $\begin{array}{l}0.1015 \\
(0.000) \\
\end{array}$ & $\begin{array}{l}0.2473 \\
(0.000) \\
\end{array}$ & $\begin{array}{l}0.1133 \\
(0.000) \\
\end{array}$ & $\begin{array}{l}0.0262 \\
(0.000) \\
\end{array}$ \\
\hline $\mathrm{N}^{\circ}$ Obs. & 279 & 279 & 279 & 276 & 279 \\
\hline \multirow[t]{2}{*}{ Adjusted-R2 } & 0.963 & 0.861 & 0.806 & 0.955 & 0.843 \\
\hline & \multicolumn{5}{|c|}{ Crecimiento de la productividad laboral $^{2}$} \\
\hline Trabajo & - & - & - & - & - \\
\hline Capital $(\beta 1)$ & $\begin{array}{l}0.3428 \\
(0.000) \\
\end{array}$ & $\begin{array}{l}0.5290 \\
(0.000) \\
\end{array}$ & $\begin{array}{l}0.5392 \\
(0.000) \\
\end{array}$ & $\begin{array}{l}0.0150 \\
(0.493) \\
\end{array}$ & $\begin{array}{l}0.1284 \\
(0.001) \\
\end{array}$ \\
\hline$T I C(\beta 2)$ & $\begin{array}{l}0.0766 \\
(0.000) \\
\end{array}$ & $\begin{array}{l}0.1419 \\
(0.000) \\
\end{array}$ & $\begin{array}{l}0.2846 \\
(0.000) \\
\end{array}$ & $\begin{array}{l}0.0888 \\
(0.000) \\
\end{array}$ & $\begin{array}{l}0.0206 \\
(0.001) \\
\end{array}$ \\
\hline $\mathrm{N}^{\circ}$ Obs. & 286 & 286 & 286 & 283 & 286 \\
\hline Adjusted-R2 & 0.840 & 0.800 & 0.743 & 0.720 & 0.378 \\
\hline
\end{tabular}

${ }^{1}$ Estimación del modelo descrito en la ecuación (3)

${ }^{2}$ Estimación del modelo descrito en la ecuación (4)

Estimación datos de panel con efectos aleatorios, no se muestran los coeficientes de la constante.

Entre paréntesis el p-valor del estadístico $t$.

Fuente: Elaboración propia con datos EUKLEMS.

En cuanto a las diferencias sectoriales observables, las estimaciones de la tabla 3 corroboran en su mayoría los resultados obtenidos mediante técnicas más convencionales, y que se han detallado en la sección anterior. En primer lugar, puede observarse como el efecto relativo de las TIC sobre el crecimiento de la producción (aproximado mediante su coeficiente $\gamma_{3}$ ) es mayor en los servicios de mercado (0.113) que en las manufacturas (0.101), especialmente en el sector de las comunicaciones (0.2473), donde además se observa como la contribución del 
factor trabajo es mucho menor que en el resto de servicios, mucho más intensivos en mano de obra.

Por lo que respecta al efecto del capital TIC sobre el crecimiento de la productividad laboral, las estimaciones obtenidas mediante el modelo de efectos aleatorios, anteriormente descrito, ponen de manifiesto dos hechos fundamentales. En primer lugar, el efecto conjunto de la capitalización es mayor en las manufacturas que en los servicios, si exceptuamos el caso de las comunicaciones. Sin embargo, si observamos el coeficiente relacionado con el capital TIC $\left(\beta_{2}\right)$ en relación con el del resto de capital $\left(\beta_{1}\right)$ puede observarse como el efecto del capital TIC sobre el crecimiento de la productividad en los servicios de mercado es mayor (0.088) que el del resto del capital (0.015), aunque no ocurre lo mismo cuando analizamos el caso de los servicios no destinados a la venta. Finalmente, como ocurría en el análisis basado en la contabilidad del crecimiento, son las comunicaciones el sector de servicios que mayor impacto presenta del capital TIC, tanto en términos de crecimiento del valor añadido, como en términos de crecimiento de la productividad laboral.

Por lo tanto, se puede llegar a la conclusión de que las externalidades del capital TIC puede ser una importante fuente de crecimiento económico en los sectores usuarios de ese tipo de capital. La posterior descomposición del crecimiento de la productividad laboral indica que la capitalización TIC y sus efectos externos son efectivamente una importante fuente de crecimiento de dicha productividad en el período considerado y para los países analizados (en la línea de otros trabajos, entre otros, Mas y Quesada, 2006; Jorgenson y Stiroh, 2000; Oliner y Sichel, 2000; Whelan, 2000, o Gordon, 2000), caracterizado, por otra parte, por una notable inversión tecnológica en todas las economías consideradas.

\section{CONSIDERACIONES FINALES}

En resumen, el conjunto de evidencias tiende a refutar la tesis tradicional sobre la baja contribución de los servicios al crecimiento agregado de la productividad. El sector en conjunto presenta crecimientos de la productividad modestos, en relación con otros sectores económicos. Pero este resultado se matiza o limita cuando el análisis se hace de forma desagregada. Existen actividades de servicios, principalmente aquellas relacionadas con las TIC y con mayor facilidad para la tecnificación y computerización, cuyo crecimiento de la productividad es considerable, dada además la intensa creación de empleo que se ha observado en algunos de ellos. Igualmente, el componente de innovación juega un papel principal en este tipo de actividades. Por lo tanto, la llamada 
"enfermedad de Baumol" parece limitarse a los servicios de consumo, mientras que no consigue explicar más que una pequeña parte de la evolución de los servicios a la producción (Maroto, 2009). Finalmente, parte de los comportamientos modestos, tanto en la productividad aparente como en la productividad total de los factores, en algunos servicios podría explicarse por impactos de la innovación que no se traducen inmediatamente o cuyo impacto relativo es menor que en otro tipo de actividades.

En cuanto a las dos primeras preguntas de investigación, los datos muestran que el crecimiento del valor añadido en los países europeos (UE-10 en este trabajo) se incrementó a partir de 1995, principalmente debido a la mayor contribución del factor trabajo, tanto por el mayor número de horas trabajadas como por el incremento en las cualificaciones de la mano de obra durante este período. La contribución del capital a dicho crecimiento no ha cambiado mucho respecto al período inmediatamente anterior, aunque sí lo ha hecho la distribución del mismo, fundamentalmente hacia aquellas actividades intensivas en el uso de capital TIC (aunque todavía lejos del avance experimentado por Estados Unidos en esta materia). Sin embargo, el factor que más ha influido en el relativamente peor comportamiento de las economías europeas con respecto a Estados Unidos a partir de mediados de los 90s ha sido la tendencia decreciente observada en el crecimiento de la productividad multifactorial en Europa, particularmente en algunos países, como España o Italia, y en algunas actividades de servicios, como la hostelería, los servicios inmobiliarios y servicios a empresas o algunos servicios no destinados a la venta, como la educación.

En cuanto a la tercera de las preguntas de investigación mencionadas al principio del trabajo, parece que el comportamiento en el sector servicios, $\mathrm{o}$ al menos en algunas de sus principales ramas económicas, ha sido uno de los factores principales a la hora de explicar el proceso de divergencia en materia de productividad entre Europa y Estados Unidos en los últimos años. Sin embargo, la evidencia mostrada en este trabajo, así como en otros recientes, muestra que esta afirmación general debe, al menos matizarse, debido a la gran heterogeneidad que presenta el sector servicios. Tanto a través del método de la contabilidad del crecimiento como de estimaciones dinámicos mediante datos de panel, los resultados de este trabajo muestran que el capital relacionado con las TIC tiene una notable contribución al crecimiento del valor añadido y de la productividad laboral. Y es en aquellas actividades donde el uso de este tipo de capital es más intensivo, incluidas algunos servicios de mercado, como el notorio ejemplo de las comunicaciones, donde se observa una tendencia positiva en la evolución de la productividad. 
Por lo tanto, la principal conclusión que se puede sacar de este análisis es que en Europa, y en especial en algunos países como el nuestro, los beneficiosos efectos de la capitalización relacionada con las TIC sobre el crecimiento de la productividad, principalmente la multifactorial, todavía no se están observando en la misma medida que en otras economías, como la estadounidense. El relativo retraso en la incorporación de este tipo de capital puede ser una de las causas. Pero no hay que olvidar también el papel de algunos problemas estructurales, como el peor nivel de cualificaciones o la estructura productiva en algunos países, a la hora de explicar este fenómeno. Sin embargo, el hincapié debe hacerse en este tipo de actividades relacionadas con las nuevas tecnologías, ya que la evidencia muestra claramente sus efectos positivos sobre el crecimiento de la productividad.

Por último, estos resultados deben ser tomados con la habitual cautela, en primer lugar, debido a la propia limitación que surge del uso de una muestra geográfica y horizonte temporal concretos. Segundo, porque el papel del capital tecnológico sobre el crecimiento de la productividad se ha analizado sin tener en cuenta otros posibles factores explicativos de dicha evolución o el papel de otro tipo de innovaciones, ajenas a la mera tecnología, como las innovaciones organizacionales o de servicio, muy importantes en el sector que nos ocupa en este trabajo.

\section{REFERENCIAS BIBLIOGRÁFICAS}

Abramovitz, M. (1956): "Resource and Output Trends in the United States since 1870", American Economic Review, Papers \& Proceedings, 5-23.

Ahmad, N., Lequiller, F., Marianna, P., Pilat, D., Schreyer, P. y Wölfl, A. (2003): "Comparing labour productivity growth in the OECD area. The role of measurement", STI Working Paper Series, 14, OCDE, París

Armstrong, P., Harchaoui, T.M., Jackson, C. y TARKhani, F. (2002): “A Comparison of Canada-US Economic Growth in the Information Age: The Importance of Investment in Information and Communication Technologies", StatisTIC Canada Working Paper 1F0027MIE, 001 , Ontario

ArRow, K. (1962): "The Economic Implications of Learning-By-Doing”, Review of Economic Studies, 29, 155-73.

Attewell, P. (1994): "Information, Technology and the Productivity Paradox", en Harris, D. (ed.) Organizational Linkages: Understanding the productivity paradox, Washington DC: National Academy Press. 
Baily, M. y Lawrence, R. (2001): "Do we have a New E-conomy", American Economic Review, 91, 308-312.

BAUMoL, W. (1967). Macroeconomics of unbalanced growth: The anatomy of urban crisis. American Economic Review, 57(3), 416-26.

Berndt, E. y Morrison, C. (1992): "High Tech Capital, Economic Performance and Labor Composition in U.S. Manufacturing Industries: an Exploratory Analysis", NBER Working Paper 4010

Billón, M., LeRA, F. y OrTiZ, S. (2007): "Evidencia del impacto de las TIC en la productividad de la empresa. ¿Fin de la paradoja de la productividad?”, Cuadernos de Economía, 30(82): $5-36$

BLS, Bureau of Labor Statistics (1997). BLS Handbook of methods, US Department of Labor Statistics, Washington DC: US Government Printing Office

BosworTH, B. y Triplett, J. (2007): “The early $21^{\text {st }}$ century US productivity expansion is still in services", International Productivity Monitor, 14, Primavera, 3-19

BRYNJOLfSSON ,E. (1993): "The Productivity Paradox of Information Technology: Review and Assessment", Communications of the ACM, Diciembre

- y Hitr, L (1996): "Paradox lost? Firm-level Evidence on the Returns to Information Systems", Management Science, 42(4), 541-58.

- (1998): "Information technology and organizational design: Evidence from microdata", Stanford and Wharton Working Paper, Massachusetts Institute of Technology, Boston

CAselli, F (1999): "Technological Revolutions", American Economic Review, 89(1), 80-102.

Cette, G. Mairesse, J. y Kocoglu,Y (2002): "Croissance économique et diffusion des TIC : le cas de la France sur longue période (1980-2000)", Revue française d'économie, 16(1), 155-92.

Colecchia, A. y SchreYer, P. (2001): "ICT Investment and Economic Growth in the 1990s: Is the United States a Unique Case?", OECD STI Working Paper, 2001/7, OCDE, Paris

Crespi, G., Criscuole, C., Haskel, J., y Hawkes, D. (2006): 'Measuring and Understanding Productivity in UK Market Services', Oxford Review of Economic Policy, 22(2), 186-202.

CUADRADO, J.R. (2004): "Tres preguntas en torno a los servicios: crecimiento, empleo y productividad”, Papeles de Economía Española, 100, 211-237.

CEA, Council of Economic Advisers (2000): Economic Report of the President \& Annual Report of the Council of Economic Advisers, Washington: US Government Printing Office.

- (2001): Economic Report of the President \& Annual Report of the Council of Economic Advisers, Washington: US Government Printing Office

Crafts, N (2002): "The Solow Productivity Paradox in Historical Perspective", CEPR Discussion Paper, 3142, London School of Economics, Londres 
Crafts, N y O’Mahony, M (2001): “A Perspective on UK Productivity Performance”, Fiscal Studies, 22, 3: 271-306.

David, P (1990): "The Dynamo and the Computer: An Historical Perspective on the Modern Productivity Paradox”, American Economic Review, 80(2): 355-61.

- (1991): "Computer and Dynamo: The Modern Productivity Paradox in a Not-too- Distant Mirror", en OECD: Technology and Productivity: The Challenge for Economic Policy, 315 48, Paris: OCDE

- (2000): 'Understanding Digital Technology's Evolution and the Path of Measured Productivity Growth: Present and Future in the Mirror of the Past', en Brynjolfsson y Kahin (eds.) Understanding the Digital Economy, Cambridge MA: MIT Press.

De Masi, P (2000): "Does the Pickup in Productivity Growth Mean That There is a 'New Economy'?’, United States of America - Selected Issues, IMF Staff Country Report No. 00/112, Washington, D.C.: International Monetary Fund.

DIEWERT, E. y Fox, K. (1999): "Can measurement error explain the productivity paradox?", Canadian Journal of Economics, 32(2): 251-280

Dosi, G. PavitT, K. y SoEte, L. (1990): The Economics of Technological Change and International Trade, Wheatsheaf, Brighton.

ELDERIDGE, L. (1999). How price indexes affect BLS productivity measures. Monthly Labor Review, 122(2), 35-46.

EPKE (2002): 'Evaluating the impact of new economy on economic performance. A review', mimeo, EPKE Research Group, Amsterdam

Fourastie, J. (1949). Le grand espoir du XXe siecle. Paris: Presses Universitaires de France.

Freeman, C. (1987): 'Information Technology and Change in Technoeconomic Paradigm', en Freeman, C. y Soete, L. (eds.), Technical Change and Full Employment, Oxford, Basil Blackwell.

- Y SoEte, L. (1997): The Economics of Industrial Innovation, Pinter, London.

Gadrey, J. y Gallouj, F. (Eds.) (2002): Productivity, innovation and knowledge in services, Edward Elgar, Cheltenham

Gallou, F. (2002a): "Innovation in services and the attendant old and new myths". Journal of Socioeconomics, 31(2), 137-154.

- (2002b): Innovation in the Service Economy. Cheltenham: Edward Elgar

Gómez, N., LóPez, L.A. y TobarRa, M.A. (2006): "Difusión y absorción de las TIC en la economía española”, Economía Industrial, 360: 117-130

Gordon, R (2000): 'Does the 'New Economy' Measure up to the Great Inventions of the Past?', Journal of Economic Perspectives, 14(4): 49-74. 
GoRDON, R (2001): 'Technology and Economic Performance in the American Economy', Department of Economics, Northwestern University, mimeo.

GRILICHES, Z. (1973): "Research Expenditures and Growth Accounting", en Williams, B. (ed.) Science and Technology in the Economic Growth, McMillan, London.

- (1979): "Issues in Assessing the Contribution of Research and Development to Productivity Growth", Bell Journal of Economics, 10, 91-116.

- (1992): Output measurement in the service sector, University of Chicago Press for NBER, Chicago

- (1994): "Productivity, R\&D and the data constraint", American Economic Review, 84(1), $1-23$

- (1995a): "The Discovery of the Residual: An Historical Note", NBER Working Paper, 5348.

- (1995b): "R\&D and Productivity: Econometric Results and Measurement Issues", en Stoneman, P. (ed.) Handbook of the Economics of Innovation and Technological Change, Basil Blackwell, Oxford.

Hernando, I. y NuÑEz, S. (2002): "The contribution of ICT to economic activity: A growth accounting exercise with Spanish firm-level data", Investigaciones Económicas, 28(2): 315348

Hernansanz, C., Melguizo, A. y Sebastián, M. (2001): "Las tecnologías de la información y la comunicación en España”, Información Comercial Española, 793: 25-38

INKLAAR, R., O'MAHONY, M. y TIMMER, M. (2005): "ICT and Europe's productivity performance industry-level growth account comparisons with the United States", GGDC Memorandum, 68, Groningen Growth and Development Centre, Groningen

JoRGenson D. W. (2001): "Information Technology and the US Economy", American Economic Review, 91(1), 1-32.

- Gollop, F.M. y Fraumeni, B. (1987): Productivity and US Economic Growth, Cambridge MA, Harvard University Press.

Jorgenson, D., Mun, H. y Stiroh, K. (2005) Information technology and the American growth resurgence, MIT, Cambridge, MA

Jorgenson, D.W. y STIROH, K. (1995): “Computers and Growth", Economics of Innovation and New Technology, 3(3-4), 295-316.

- (2000): 'Raising the Speed Limit: U.S. Economic Growth in the Information Age', Brookings Papers on Economic Activity, 1: 125-211.

Lebow, D., y RudD, J. (2003). Measurement error in the consumer price index. Where do we stand?. Journal of Economic Literature, 41(1), 159-201.

Loveman, G. (1988): "An Assessment of the Productivity Impact of Information Technologies", Sloan School of Management, MIT, Working Paper 90S-88-054. 
LuCAs, R. E. (1988): “On the Mechanics of Economic Development”, Journal of Monetary Economics, 22, 3-42.

McLachlan, R., ClaRK, C. y Monday, I. (2002): ‘Australia's service sector: A study in diversity', Productivity Commission Staff Research Paper, AusInfo, Canberra

MAROTO, A. (2009a): La productividad en el sector servicios de la economía española, Colección Economía y Empresa, Marcial Pons, Madrid

- (2009b): "Nuevas evidencias sobre la productividad de los servicios en la economía española”, Papeles de Economía Española, 120: 44-68

- (2010a): "Productivity in service industries: Conventional and current explanations", The Service Industries Journal (en publicación)

- (2010b): "Productivity in European private and public services: A growth accounting exercise", Journal of Service Science, 2: 25-53

MaRoto, A. y CUAdRAdo, J.R. (2006): La productividad de la economía española, Colección Estudios, Instituto de Estudios Económicos, Madrid

- (2008): "Evolución de la productividad en España. Un análisis sectorial, 1980-2006", Economía Industrial, 367, 1-21

- (2009): "Is growth of services an obstacle to productivity growth? A comparative analysis", Structural Change and Economic Dynamics, 20(4): 254-65

Maroto, A. y Rubalcaba, L. (2008): "Services productivity revisited", The Services Industries Journal, 28(3), 1-17

MAS, M. y QuesadA, J. (2005): Las nuevas tecnologías y el crecimiento económico en España, Fundación BBVA, Bilbao

- (2006) 'The role of ICT in the Spanish productivity slowdown', Documentos de Trabajo, 5, Fundación BBVA, Madrid

MAS, M. y RoBledo, J.C. (2010): Productividad: Una perspectiva internacional y sectorial, Fundación BBVA, Bilbao

Mason, G., Wagner, K., Finegold, D. y Keltner, B. (2000): "The 'IT Productivity Paradox' Revisited: International Comparisons of Information Technology, Work Organisation and Productivity in Service Industries", Vierteljahrshefte zur Wirtshaftsforschung, 69(4), 618-29

McKinsey Global Institute (2001): U.S. Productivity Growth, 1995-2000, Washington D.C.: McKinsey Global Institute.

Nelson, R. y Winter, S. (1982): An Evolutionary Theory of Economic Change, Cambridge (Mass.) Harvard University Press.

NÚÑEz, S. (2001): "Las nuevas tecnologías y su contribución al crecimiento económico español", Economía Industrial, 340: 61-72

OCDE (2001a): Innovation and productivity in services, OCDE, París 
OCDE (2001b). Measuring productivity - OECD Manual, Measurement of aggregate and industry-level productivity growth, OCDE, París

- (2005): Growth in services. Fostering employment, productivity and innovation, OCDE, París

Oliner, S. y Sichel, D. (1994): "Computers and Output Growth Revisited: How Big is the Puzzle?", Brookings Papers on Economic Activity, 2: 273-317.

- (2000): 'The Resurgence of Growth in the Late 1990s: Is Information Technology the Story ?', Journal of Economic Perspectives, 14(4), 3-22.

- (2002): 'Information Technology and Productivity: Where are we Now and Where are we Going?, Board of Governors of the Federal Reserve, Finance and Economics Discussion Paper, 2002-29.

O'Mahony, M. y DE Boer, W. (2002): Britain's Relative Productivity Performance, Updates and Extensions, National Institute of Economic and Social Research, Londres

- y Vecchi, M. (2002): Knowledge Based Capital and Economic Performance, National Institute of Economic and Social Research, Londres

- y VAN ArK, B. (2003): EU productivity and competitiveness. An industry perspective. Can Europe resume the catching-up process?, Enterprise Publications, Comisión Europea, Bruselas

Oulton, N. (2001): 'Must the growth rate decline? Baumol's unbalanced growth revisited', Oxford Economic Papers, 53, 605-27.

PAVITT, K 1984. Sectorial patterns of technical change: towards a taxonomy and a theory. $R e$ search Policy, 13, 343-373Petit, P. y Soete, L. (2001): Technology and the Future of European Employment, Edward Elgar, Cheltenham.

Perez, C. (1983): 'Structural Change and the Assimilation of New Technologies in the Economic and Social System', Futures, 15(3), 357-75.

- (1985): 'Microeletronics, Long Waves and World Structural System: New Perspectives for Developing Countries', World Development, 13, 441-463.

Pérez, F., Maudos, J., Pastor, J.M. y Serrano, L. (coords.) (2006): Productividad e internacionalización: el crecimiento español ante los nuevos cambios estructurales, Fundación BBVA, Madrid

PILAT, D. (2001): "Productivity growth in ICT-producing and ICT-using industries: A source of growth differentials in the OECD?", OECD STI Working Paper, 2001/04, OCDE, París

- (2004): 'The ICT productivity paradox. Insights from microeconomic data', OECD Economic Studies, 38, Paris: OECD.

RoACH, S. (1991): 'Services Under Siege - The Restructuring Imperative', Harvard Business Review, Septiembre-Octubre, 82-92

Romer, P. (1990): 'Endogenous Technological Change', Journal of Political Economy, 98, S71S102. 
Rubalcaba, L. (2007). The new service economy. Cheltenham: Edward Elgar.

SCHREYeR, P. (1998): 'Information and Communication Technology and the measurement of real output, final demand and productivity', STI Working Papers 1998/2, Paris: OCDE.

- (2000): 'The Contribution of Information and Communication Technology to Output Growth: A Study of the G7 Countries', OECD STI Working Paper, 2000/2, París: OCDE

SEgARRA, A. (2010): "Innovation and productivity in manufacturing and service firms in Catalonia: A regional approach", Economics of Innovation and New Technology, 19(3): 233-58

Siegel, D. (1994): 'Errors in Output Deflators Revisited. Unit Values and the Producer Price Index', Economic Inquiry, 32, 11-32.

- (1997): 'The Impact of Computers on Manufacturing Productivity Growth: A Multiple-Indicators, Multiple-Causes Approach," Review of Economics and StatisTIC, 68-78.

SilverberG, G. y Verspagen, B. (1994): 'Learning, Innovation and Economic Growth: A Longrun Model of Industrial Dynamics', Industrial and Corporate Change, 3(1), 199-223.

Soete L. y Turner, R. (1984): 'Technology Diffusion and the Rate of Technical Change', Economic Journal, 94, 612-23.

Solow, R. (1957): 'Technical Change and the Aggregate Production Function', Review of Economics and StatisTIC, 38, 312-20.

STIROH, K. (1999): 'Is there a New Economy?', Challenge, 42(4), 82-101.

- (2001): 'Investing in Information Technology: Productivity Payoffs for U.S Industries', Current Issues in Economics and Finance, 7, 6.

- (2002), 'Are ICT Spillovers Driving the New Economy?', The Review of Income and Wealth, $48,33-57$

Strassman, P. (1985), Information Payoff, New York: Free Press.

Triplett, J. (1999): 'The Solow Productivity Paradox: What do Computers do to Productivity?', Canadian Journal of Economics, 32(2), 309-34

- y Bosworth, B. (2004): Productivity in the U.S. Services Sector: New Sources of Economic Growth. Washington, D.C.: Brookings Institute

Timmer, M., O'MAHONY, M. y van ARK, B. (2008) 'EU KLEMS growth and productivity accounts. Overview November 2008 release', mimeo, Groningen Growth and Development Centre, Groningen

TrIPlett, J. y Bosworth, B. (2003): "Productivity measurement issues in service industries: Baumol's disease has been cured", Economic Policy Review, Federal Reserve Bank of New York, Septiembre

- (2005): Productivity in the U.S. service sector. New sources of economic growth, Brookings Institution Press, Washington D.C.

UPPENBERG, K. y STRAuss, H. (2010): Innovation and productivity in the EU services sector, Economic and financial studies, European Investment Bank, Luxemburgo 
UzAWA, H. (1965): 'Optimal technical change in an aggregative model of economic growth', International Economic Review, 6, 18-31.

VAN ARK, B. (1996): "Issues in productivity measurement: statistical problems and policy links", en OCDE: Industry productivity: International comparisons and measurement issues, 19-47, OCDE, París

- (2001): 'The Renewal of the Old Economy: An International Comparative Perspective', OECD STI Working Paper, 2001/5, OCDE, París

VAN ARK, B., Broersma, L. y de Jong, G. (1999): 'Innovation in Services. Overview of Data Sources and Analytical Structures', Research Memorandum GD-44, Groningen Growth and Development Centre, University of Groningen

VAN ARK, B. y INKLAAR, R. (2007): "Catching up or getting stuck? Europe's trouble to exploit ICT's productivity potential", Research Memorandum 79, Groningen Growth and Development Centre, Groningen

- y McGuckIN, R. (2002): "Changing gear. Productivity, ICT and service industries: Europe and United States", GGDC Research Memorandum 60, Groningen Growth and Development Centre, Groningen

- (2003): ICT and productivity in Europe and the United States. Where do the differences come from?, Groningen Growth and Development Centre, University of Groningen

VijselaAr F. y Albers R. (2002): 'New Technologies and Productivity Growth in the Euro Area', Working Paper Series, 122, European Central Bank, Frankfurt.

Whelan, K. (2000): 'Computers, Obsolescence and Productivity', Federal Reserve Board Finance and Economics Discussion Paper Series, 2000-6.

WIEL, H.P. van der (2001): "Does ICT boost Dutch productivity growth?", CPB Documents, 16, CPB Netherlands

WolfF, E. (1999): "The productivity paradox: evidence from indirect indicators of service sector productivity growth”, Canadian Journal of Economics, 32(2), 281-308.

WöLfL, A. (2003), Productivity growth in service industries. An assessment of recent patterns and the role of measurement. STI Working Paper, 2003-7, Paris: OECD. 